\title{
Single-Event Upsets in Photoreceivers for Multi-Gb/s SLHC Data Transmission
}

Sarah Seif El Nasr-Storey, Stephane Detraz, Ping Gui, Mohsine Menouni, Paulo Moreira, Spyridon Papadopoulos, Christophe Sigaud, Csaba Soos, Pavel Stejskal, Jan Troska, and Francois Vasey

\begin{abstract}
A $63 \mathrm{MeV}$ proton beam was used to perform a single event upset (SEU) test on a candidate component for a future high luminosity large hadron collider (HL-LHC) high speed optical. An in-lab error injector was used to show that 1-0 bit errors are caused by the amplifier's response to the large signal caused by a single event transient (SET) in the photodiode.
\end{abstract}

Index Terms-High speed optical links, LHC, single event upsets.

\section{INTRODUCTION}

$\mathbf{T}$ HE large hadron collider (LHC) [1], a proton-proton collider at the European Organization for Nuclear Research (CERN, Geneva, Switzerland), has been in operation since the fall of 2009. The four experiments at the LHC were the first in High Energy Physics (HEP) to make extensive use of high speed optical data transmission links. Plans to increase, by an order of magnitude, the luminosity of the LHC after ten years to $10^{35} \mathrm{~cm}^{-2} \mathrm{~s}^{-1}$ will improve the experiments' chances of observing the rare processes they are searching for, as well as increasing both the amount of data transmitted and the radiation doses received by the links. This will impose even more stringent requirements on the performance and radiation tolerance of the optical transmission links used in these systems.

Currently, each LHC experiment has developed specific data transmission links for the data acquisition (DAQ), slow control (SC), and timing and trigger distribution (TTC) systems of their detectors. The Versatile link project [2] aims to develop the optoelectronic components required to build a fast, bidirectional, radiation hard optical link which can serve all three transmission applications; while the gigabit transceiver (GBT [3]) project aims to develop the chipset required to de/serialize and de-/encode the transmitted data. The part of the link located inside the detector volume, the versatile transceiver (VTRx), is

Manuscript received July 22, 2011; revised September 08, 2011 and October 07, 2011; accepted October 08, 2011. Date of publication November 23, 2011; date of current version December 14, 2011.

S. Seif El Nasr-Storey, S. Detraz, P. Moreira, S. Papadopoulos, C. Sigaud, C. Soos, P. Stejskal, J. Troska, and F. Vasey are with CERN, CH-1211, Geneva, Switzerland (e-mail: Sarah.Storey@cern.ch; Stephane.Detraz@cern.ch; Paulo.Moreira@cern.ch; Spyridon.Papadopoulos@cern.ch; Christophe.Sigaud@cern.ch; Csaba.Soos@cern.ch; Pavel.Stejskal@cern.ch; Jan.Troska@cern.ch; Francois.Vasey@cern.ch).

P. Gui is with Southern Methodist University, Dallas, TX, 75205 USA (e-mail: pgui@1yle.smu.edu).

M. Menouni is with CPPM, 13288 Marseille, France (e-mail: menouni@cppm.in2p3.fr).

Color versions of one or more of the figures in this paper are available online at http://ieeexplore.ieee.org.

Digital Object Identifier 10.1109/TNS.2011.2172632 expected to operate (error free) in a radiation environment dominated by high energy pions and where particle fluxes can reach $10^{8} \mathrm{~cm}^{-2} \mathrm{~s}^{-1}$. A prototype of the receiver has been made which contains a commercial $60 \mu \mathrm{m}$ InGaAs photodiode packaged into a receiver optical subassembly (ROSA) with the gigabit transimpedance amplifier (GBTIA [4]): a custom-designed receiver amplifier that is part of the GBT chipset.

Several concerns have been raised regarding the operation of the GBTIA ROSA in the upgraded LHC environment. The first is that single event upsets (SEUs) are known to occur in photodiodes placed in similar radiation environments [5]-[7]. Secondly, previous work [5], [7], [8] has shown that SEUs can produce multiple-bit upsets (bursts) in optical receiver. Pacheco et al. [8] tried to understand the statistics of the different types of errors occurring in receivers, and showed that bursts of lengths up to hundreds of bits long can be produced in ROSAs placed in a proton beam. These bursts, were they to occur in the GBTIA ROSA, would be too long for the forward error correction (FEC) algorithm employed in the GBT protocol to correct. The GBT's FEC algorithm can correct a maximum of 16 errors per frame of 120 bits. For these two reasons, and as part of the qualification process of the GBTIA ROSA prototype, an SEU test was carried out using the low energy proton irradiation facility (PIF-NEB [9]) at the Paul Scherrer Institute (PSI), Villigen, Switzerland.

In-beam testing is almost always a necessity when investigating the SEU performance of optoelectronic components for use in harsh radiation environments. Such tests are expensive, time-consuming, and usually difficult to carry out, so we designed an in-lab error simulator to reproduce some of the singleevent effects observed during an in-beam test. This error injector was also used to extend the work done in the papers mentioned above and provide a method for understanding the origins of different types of errors in the lab. This paper discusses the results obtained from both the proton beam SEU test and what was understood using the in-lab error simulator.

\section{EXPERIMENTAL PROCEDURE}

The choice of proton facility was made to fulfill the primary aim of the test which was to compare the performance of the custom designed receiver with both a commercial receiver and devices previously tested [8] in similar radiation environments. A more representative test, in terms of both particle species and energy, may have to be performed to measure the receiver's performance in its intended environment, which is dominated by high energy pions. 


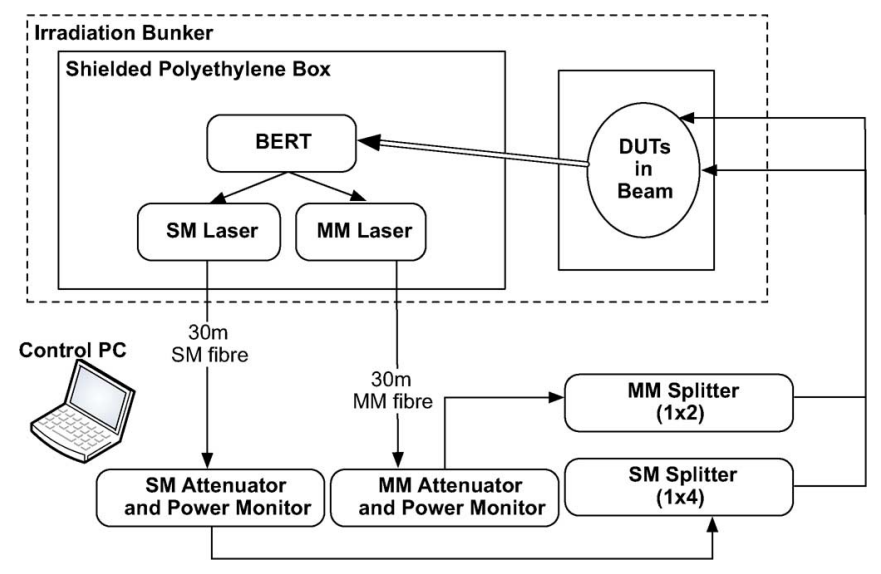

Fig. 1. Experimental set up used for the proton irradiation test.

TABLE I

DEVICES UNDER TEST: DUTS

\begin{tabular}{ccc}
\hline \hline Family & Active Diameter & Amplifying Components \\
\hline PIN SM & $60 \mu \mathrm{m}$ & commercial TIA+LA \\
GBTIA ROSA & $60 \mu \mathrm{m}$ & GBTIA \\
MM ROSA & $80 \mu \mathrm{m}$ & commercial TIA+LA \\
\hline \hline
\end{tabular}

\section{A. Irradiation Set-Up}

The irradiation test took place in July 2010, at the PIF-NEB facility at PSI using a bunched $63 \mathrm{MeV}$ proton beam. The beam was pulsed with a repetition frequency of $51 \mathrm{MHz}$, with each proton bunch lasting less than a nanosecond. The proton flux was $90 \%$ uniform over a radius of approximately $5 \mathrm{~cm}$ thus allowing the arrangement of six photodiodes within the beam spot to be tested simultaneously. The test was carried out at a flux of approximately $2 \times 10^{8} \mathrm{~cm}^{2} \mathrm{~s}^{-2}$, equivalent to four protons per bunch and square centimeter. Ionization chambers located behind the beam exit window measured and recorded the flux every second.

Data were generated, and errors recorded, by a multichannel FPGA-based bit error rate tester (BERT) [10] placed in a shielded box below the beam axis. The box was made from a combination of Polyethylene and Aluminum of sufficient thickness to stop the $63 \mathrm{MeV}$ protons used to irradiate the devices. The serial data was then fed to a laser driver and laser diode for transmission to the devices placed in the beam line (see Fig. 1). Optical fibers, $30 \mathrm{~m}$ in length, were used to send the optical signals to the control room where their amplitudes were monitored and controlled via optical power monitors and splitters.

\section{B. Devices Tested}

The devices were selected to provide a comprehensive comparison between the GBTIA ROSA and a commercial ROSA. The SM p-i-n was included in the test to allow for comparison with the previous survey of devices performed at CERN in 2007. Table I shows a complete list of the devices tested. Six devices, two of each type, were irradiated in total.

The GBTIA ROSA, the receiving component of the versatile transceiver intended for use by experiments at the HL-LHC, consists of a commercial $60 \mu \mathrm{m}$ InGaAs photodiode packaged with the GBTIA. The GBTIA is a $5 \mathrm{~Gb} / \mathrm{s}$, fully differential and highly sensitive optical receiver designed and implemented in a commercial $0.13 \mu \mathrm{m}$ CMOS process. The GBTIA chip consists of a low-noise, high bandwidth transimpedance amplifier (TIA) and a high performance limiting amplifier (LA) followed by a $50 \Omega$ output stage. A novel biasing circuit is designed in the TIA to cope with the potentially high leakage current induced in the photodiode by radiation.

Each device, along with the amplifying components required to transmit the signal over the coaxial cables to the shielded BERT, was mounted on a small test board. The active devices on each board were shielded from the proton beam by $6.5 \mathrm{~mm}$ of brass; except in the case of the ROSAs, where the TIAs were also exposed to the incident protons by virtue of the fact that they were housed in the same optical subassembly as the photodiodes.

The test boards were then arranged on a frame so that all six devices were in the beam spot, and the frame was placed on a rotating axle that allowed the angle of incidence between the devices and the beam to be varied between $0^{\circ}$ and $180^{\circ}$. Previous work [8], [11] has demonstrated that optical fibers and connectors can partially shield DUTs from the incident particles. To avoid any "accidental" shielding of the devices by the optical fibers the frame was placed in the beam line so that the underside of the DUTs faced the beam, i.e., there was no excess material between the incident particles and the photoreceivers.

\section{Bit Error Rate Tester}

The BERT is implemented on an ML523 Transceiver characterization platform from Xilinx and supports up to six high speed transceivers-each operating at a maximum data-rate of $6.5 \mathrm{~Gb} / \mathrm{s}$. The firmware was developed so that the transmitted data-rate could be modified by changing the frequency of the base clock provided to the BERT. This enabled data to be collected at 2.0, 3.0, 4.0, $5.0 \mathrm{~Gb} / \mathrm{s}$ (the target data rate for the final application is $4.8 \mathrm{~Gb} / \mathrm{s})$. This was a wider range of data rates than that covered in the previous SEU test [8], where the highest data rate tested was $2.5 \mathrm{~Gb} / \mathrm{s}$.

A simplified schematic of the BERT's firmware implementation is shown in Fig. 2. The BERT uses the GBT protocol to de-/encode the transmitted/received data/frames, and is therefore capable of correcting up to 16 bit-errors per frame of 120 bits. Counters are implemented for each channel to record the number of:

- errors before the decoder (line errors);

- errors after the decoder (system errors);

- transmitted words.

In addition to these counters, an error log memory is implemented for each BERT channel. A new line is written to a channel's error log whenever one or more bit-errors are detected-either before or after the decoders. Each line of the error $\log$ consists of a:

- channel number;

- a time stamp corresponding to the global word counter;

- the transmitted data before and after the encoder;

- the received data before and after the decoder. 


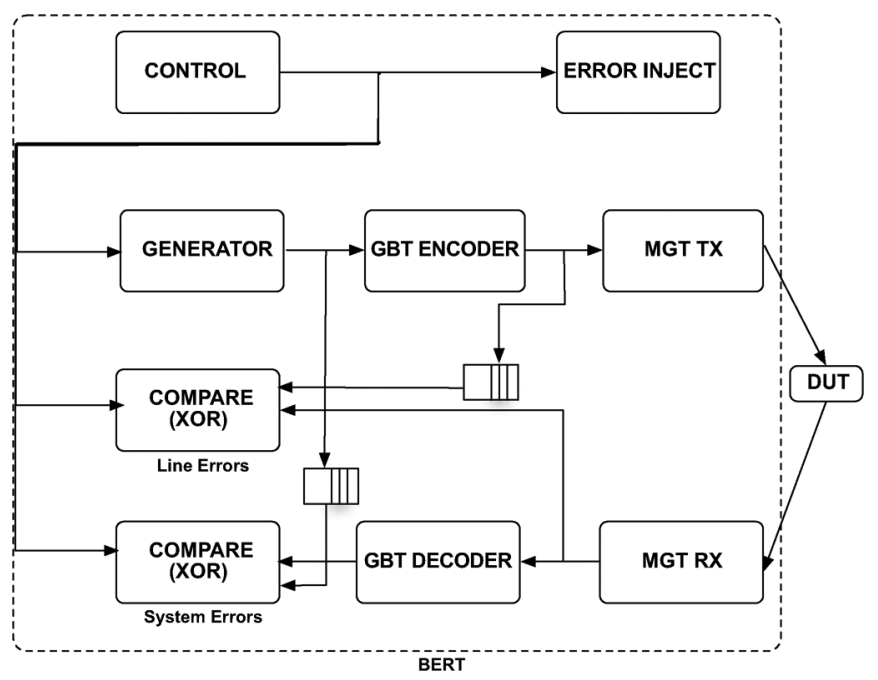

Fig. 2. Simplified schematic of the BERT's firmware implementation.

The information provided by the error logging mechanism implemented in the BERT made it possible to perform the detailed analysis of the error statistics presented here.

\section{SEU Test REsults}

Data were recorded for each device under test at each data rate $(2.0,3.0,4.0,5.0 \mathrm{~Gb} / \mathrm{s})$ and angle of incidence of the proton beam $\left(0,10,80,90,100,110^{\circ}\right)$. For all results shown in this paper, the optical modulation amplitude (OMA) is defined as the signal amplitude at the input of the device, obtained by correcting the power measured in the control room (see Fig. 1). In addition we define the bit error cross section $(\sigma)$ in terms of the data rate $(R)$, the average proton flux $(\phi)$, and the Bit Error Rate $(B E R)$ as:

$$
\sigma=\frac{\mathrm{R}^{*} \mathrm{BER}}{\phi}
$$

This cross section is only meaningful in the SEU dominated region, which is defined apriori as the region of the BER curve where the OMA exceeds that required to reach an error rate of $10^{-10}$ when the beam is off.

\section{A. Bit Error Rate Sensitivity}

The effect of the proton beam on the BER performance of the photodiodes under test is shown in Fig. 3.

When the beam is on the range of OMA can be divided into two regions. The first where the dominant cause of errors is electrical and environmental noise, and the second where most errors are caused by the incident protons.

The effect that the angle of incidence of the proton beam has on the bit error cross section is shown in Fig. 4 for the GBTIA ROSA at $5.0 \mathrm{~Gb} / \mathrm{s}$, but the results were similar for all the DUTs and all the data rates tested.

The bit error cross section is strongly dependant on the incident angle of the proton beam, and the greatest number of bit errors are induced in the device when the proton beam is at grazing incidence with respect to the DUT.

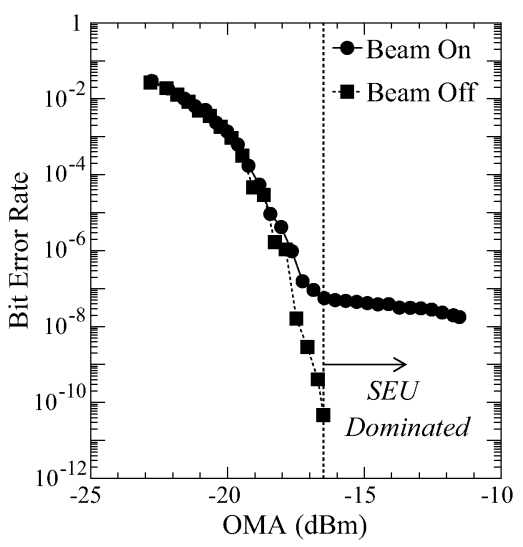

Fig. 3. Errors caused by the proton beam at grazing incidence to the devices in the GBTIA ROSA receiving data at $5.0 \mathrm{~Gb} / \mathrm{s}$.

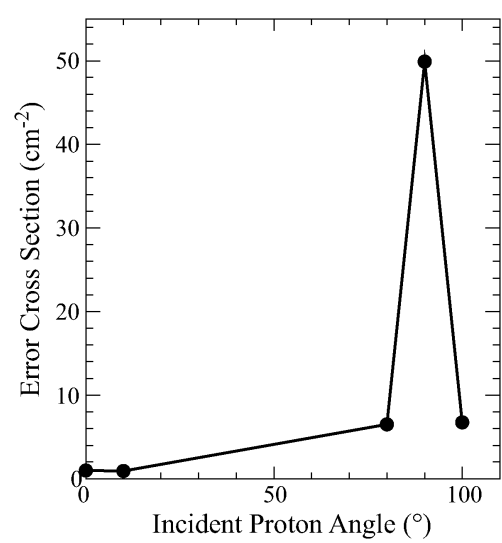

Fig. 4. Bit error cross section for the GBTIA ROSA taken at a constant OMA for all angles of incidence of the proton beam.

Previous studies [5]-[7] have shown that at low values of OMA energy deposition due to direct ionization is the dominant cause of single event transients in photodiodes, while at high values of OMA only nuclear recoils can deposit the energy required to cause errors in the receivers. Therefore the BER is dependent on the incident angle of the proton beam at low input power levels, and independent of the angle of incidence for high input power levels. Since input power levels in the devices under study were never sufficient to reach the region where the BER is independent of angle, as shown in Fig. 4, we conclude that most errors observed in the receivers were caused by direct ionization and not nuclear recoils. Fig. 4 also shows that the excess in the error cross section near normal incidence seen in previous tests [8] is no longer present, i.e. irradiating the devices with their undersides facing the beam was successful in removing any excess shielding material between the DUTs and the incident particles.

One of the aims of the SEU test presented in this paper was to compare the behaviour of the GBTIA ROSA with that of commercial receivers. In Fig. 5 we plot the bit error cross section as a function of OMA for all DUTs and a $60 \mu \mathrm{m} \mathrm{p}$-i-ns from the survey of devices performed in December 2007 (the same 60 $\mu \mathrm{m}$ p-i-n was irradiated in both tests). The devices from both tests exhibit the same general behaviour as a function of the input power at the receiver. The differences in magnitudes at 


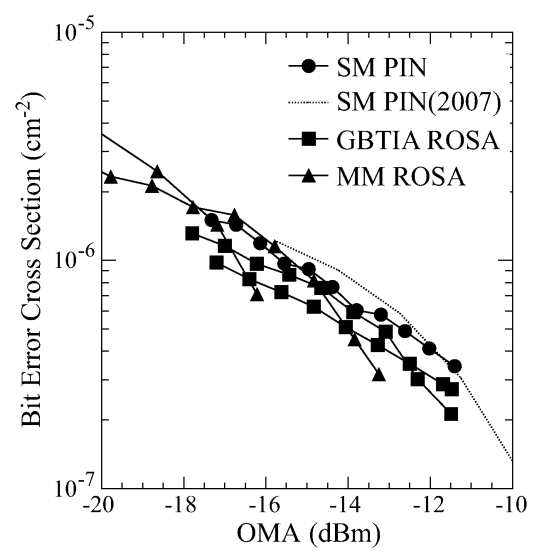

Fig. 5. All data was taken at $2.0 \mathrm{~Gb} / \mathrm{s}$ and an average flux of $2 \times 10^{8} \mathrm{p} / \mathrm{cm}^{2} / \mathrm{s}$. The data shown for the 2007 test was taken at $2.5 \mathrm{~Gb} / \mathrm{s}$ and a particle flux of $8 \times 10^{8} \mathrm{p} / \mathrm{cm}^{2} / \mathrm{s}$.

a given OMA between the two p-i-ns could be explained by the different transmission rates and the different LAs used to amplify the signals send to the BERT in the two tests. Therefore the GBTIA ROSA to SEUs is comparable to other commercially available receivers of the same active diameter.

Fig. 5 also shows that the ROSAs are no more susceptible to transients than the bare photodiodes; despite the fact that in the case of the ROSAs we are observing the effect of the proton beam on both the photodiode ant the TIA (recall both are exposed to the incident particles). We thus conclude that most transients observed are due to upsets in the photodiode and not in the TIA. This results is in agreement with previous experimental results [8].

\section{B. Burst Identification Methodology}

Previous tests [8] have shown that multibit errors (bursts) can occur in ROSAs where the amplifier is not shielded from the incident particles. The bursts previously observed were 100's of bits long; the FEC scheme implemented by the GBT protocol can only correct a maximum of 16 bits per transmitted frame. Therefore, it was an important part of this test to indentify whether these long bursts occur in the GBTIA ROSA. The error logging mechanism implemented in the BERT allowed us not only to count the number of errors induced by SEUs in the devices tested, but also to record the transmitted and received data, before and after the de-/encoder, associated with those errors. This allowed us to perform a detailed study of the error statistics using Error Free Interval (EFI) histograms, burst histograms, and the BER after the FEC scheme.

1) Burst Visualization: Two-dimensional, color coded burst length histograms are used to encode all the information about the types of bursts occurring in a device into a single, compact plot. An example of such a plot is shown in Fig. 6.

A circle is drawn at each point $(x, y)$ if at a power level $x$ there occurs one or more bursts of length $y$. The length of the burst is defined as the total number of bits in a burst. The size of the circle is logarithmically proportional to the contribution of all bursts of length $y$ to the BER at this power level. Finally, the color of the circle can represent either the:

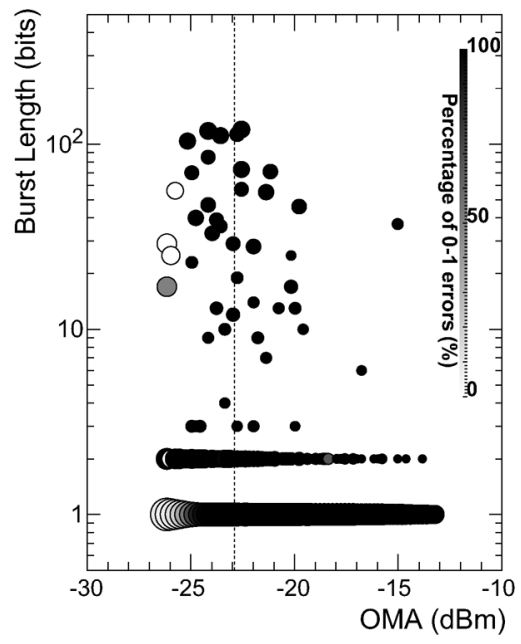

Fig. 6. Example of a 2-D, color-coded, burst length histogram for a MM ROSA at $3.0 \mathrm{~Gb} / \mathrm{s}$ and grazing incidence. The dashed line indicates the value of OMA where errors are dominated by SEUs.

- average burst occupancy: fraction of bits in error within a burst, i.e. number of bits in error in a burst divided by the burst length;

- average 0-1: fraction of bits in error within a burst due to a transmitted 1 being received as a 0 (or vice-versa).

2) Burst Identification: A method to distinguish between single bit errors and burst errors must be defined if all bit flips originated by the same physical cause are to be grouped together. A burst is defined as a group of bits in which two bits in error are separated by no more than $\mathrm{X}$ valid bits, in other words $\mathrm{X}$ is the maximum number of (successive) correct bits allowed within a burst and is called the error free threshold (EFT) [12], [13]. The space between bursts is defined as the error free interval (EFI).

The EFT is set by examining the effect of changing the EFT on the EFI histograms Fig. 7(a) and (b). Due to the bunched nature of the beam, and the small active area of the device, a histogram of the EFI at a given OMA value should be centered close to the inverse of the BER at that power level. If the EFT is chosen to be too low, as in Fig. 7(a), a significant population of the EFI appears at values much smaller than 1/BER. These disappear when the EFT is set to the correct value Fig. 7(b).

The BER before and after the FEC, shown here in Fig. 8(a) and (b), can also be used to confirm that the EFT was set correctly.

Fig. 8(a) shows that no bursts longer than 10 bits occur in the MM ROSA, i.e. all errors occurring in the device would have been corrected by the FEC. Examination of the error rate after the FEC scheme, Fig. 8(b), indicates that some of the errors occurring in the device were not corrected by the FEC. Therefore the burst histogram shown in Fig. 8(a) is misleading, and the EFT value chosen to generate it is incorrect. When the EFT is set correctly (Fig. 6) the burst histogram agrees with the results obtained by examining the BER after the FEC. In the results discussed below, the EFT was set to 75 bits for the MM ROSA and to 10 bits for all the other devices. 


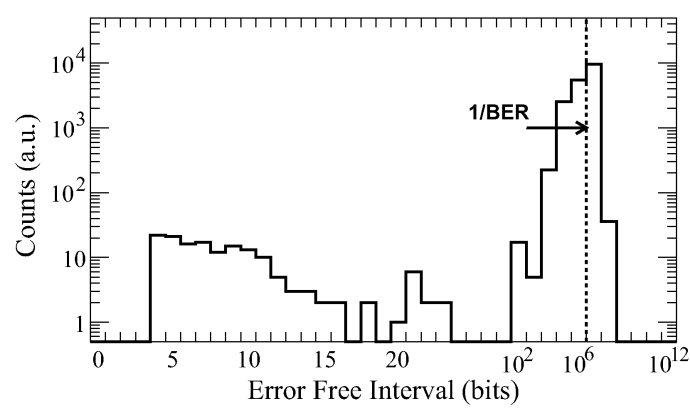

(a)

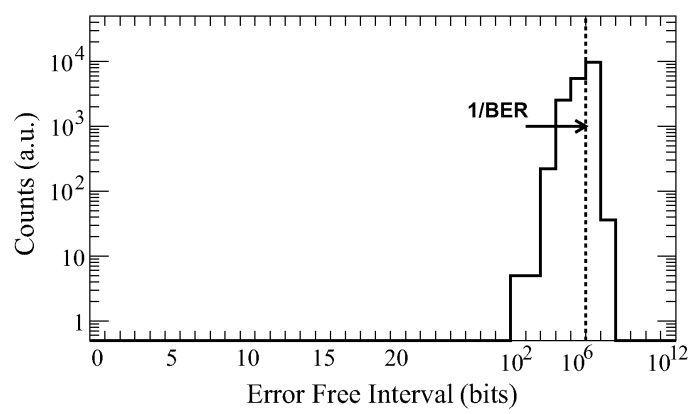

(b)

Fig. 7. MM ROSA at $5.0 \mathrm{~Gb} / \mathrm{s}$, the dashed line indicates the inverse of the BER at the power level which the histogram is drawn at. (a) EFI histogram at $\mathrm{EFT}=0$ bits. (b) EFI histogram at EFT $=75$ bits.

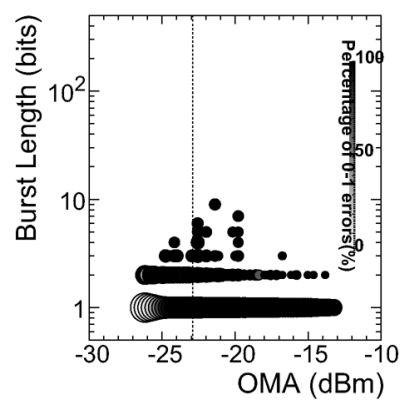

(a)

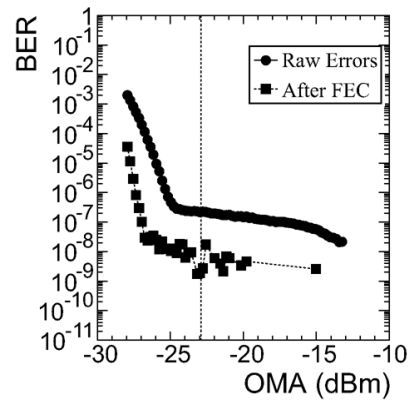

(b)
Fig. 8. MM ROSA at $5.0 \mathrm{~Gb} / \mathrm{s}$, the dashed line represents the noise level in the device. (a) Burst histogram at EFT $=0$ bits. (b) BER before and after the FEC.

\section{Burst Analysis}

The two-dimensional burst histograms described above can be used to classify the types of errors caused by SETs into three categories. These categories are:

- single bit errors;

- short bursts with a maximum burst length of 5 bits;

- long bursts with a burst length longer than 5 bits.

Fig. 9 shows a sample of the burst length histograms for all the devices tested. Only points that appear in the SEU dominated region, i.e. those that appear to the right of the dotted line in the burst histograms, are considered in this analysis. Fig. 10 shows the contributions to the total error cross section of the GBTIA ROSA from the three different categories of errors described above.

The majority of bit errors that occurred in the devices were due to 0-1 bit flips. 1-0 single bit errors were observed in all the devices tested-under all conditions-but were less frequent.
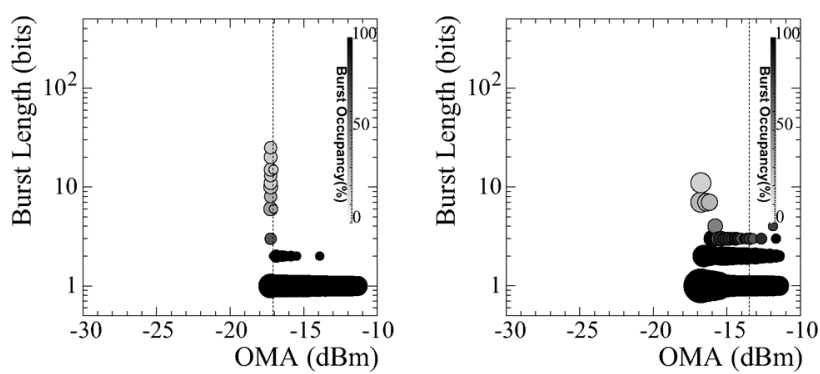

(a)
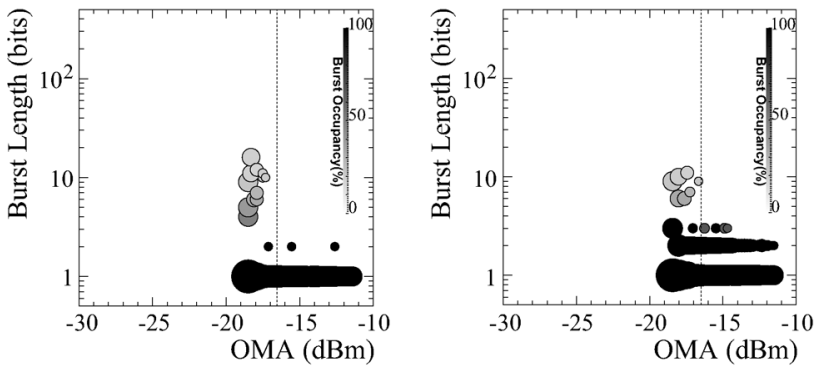

(b)
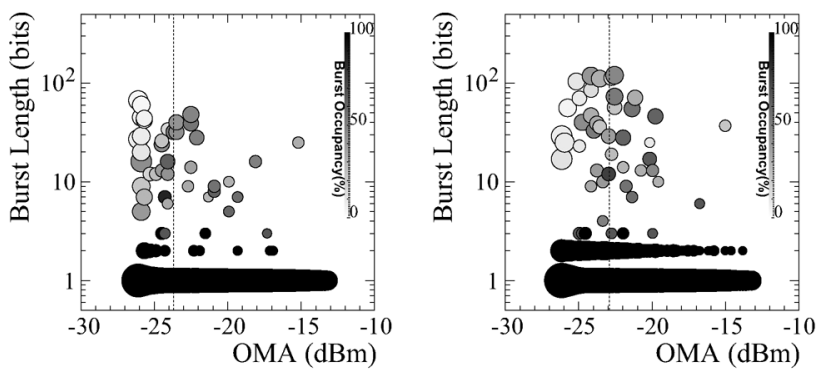

(c)

Fig. 9. Two-dimensional color coded burst length histograms for all three types of devices at $5.0 \mathrm{~Gb} / \mathrm{s}$. Once again, the SEU dominated region is that to the right of the dashed line. (a) SM PIN at $2.0 \mathrm{~Gb} / \mathrm{s}$ (LHS) and $5.0 \mathrm{~Gb} / \mathrm{s}$ (RHS). (b) GBTIA ROSA at $2.0 \mathrm{~Gb} / \mathrm{s}$ (LHS) and $5.0 \mathrm{~Gb} / \mathrm{s}$ (RHS). (c) MM ROSA at $2.0 \mathrm{~Gb} / \mathrm{s}$ (LHS) and $5.0 \mathrm{~Gb} / \mathrm{s}$ (RHS).

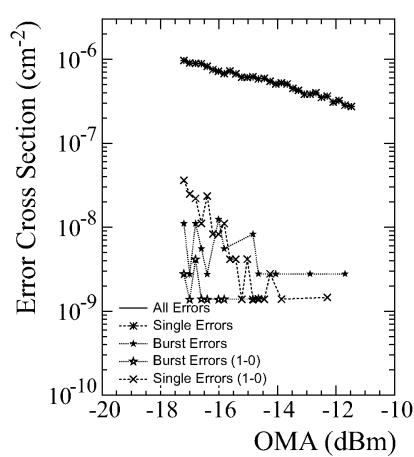

(a)

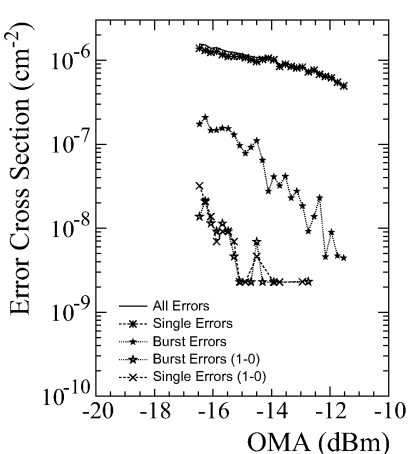

(b)
Fig. 10. Contributions to the total error cross section from the three different categories of errors. Shown here for a GBTIA ROSA at two different data rates and grazing incidence. (a) GBTIA ROSA at $3.0 \mathrm{~Gb} / \mathrm{s}$. (b) GBTIA ROSA at 5.0 $\mathrm{Gb} / \mathrm{s}$.

Single bit errors were more likely to occur at grazing incidence than at normal incidence, and at lower values of OMA. Short bursts were also found to occur in all the devices tested, and to demonstrate a dependance on the transmitted power and the angle of incidence of the proton beam similar to that of the 


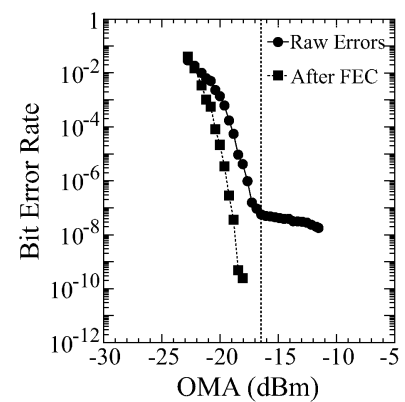

(a)

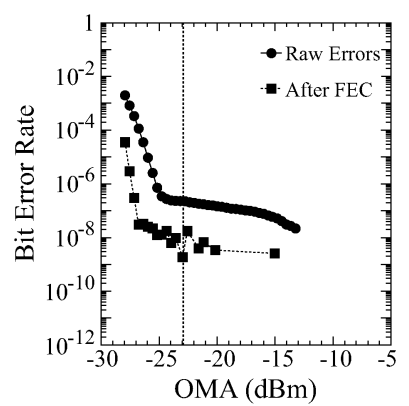

(b)
Fig. 11. GBTIA ROSA at $5.0 \mathrm{~Gb} / \mathrm{s}$ : on-line demonstration of FEC scheme's ability to correct all errors in the GBTIA ROSA. (a) GBTIA ROSA. (b) MM ROSA.

single bit errors. However, they were also found to strongly depend on the transmitted data rate. Finally, long bursts were only present in the commercial MM ROSAs. Unlike the other two categories of burst errors, which were found to have an occupancy of $100 \%$ and contain some 1-0 bit flips, long bursts were found to consist of only $0-1$ bit errors and be of very low occupancy. They were also found to be independent of transmitted power, data rate, and the angle of incidence of the proton beam.

Previously any qualification of a chosen FEC scheme's ability to mitigate bursts had to be performed offline. The BERT's implementation of the GBT's FEC scheme provided us with the opportunity to perform this qualification online. Fig. 11 shows the difference between the raw and corrected error rates for two of the DUTs.

Fig. 11 shows that all errors that occurred in the GBTIA ROSA were corrected by the FEC, whilst some occurred in the MM ROSA that could not be corrected. This is in agreement with the results shown in Fig. 9(b) and (c) which show that the longest burst occurring in the GBTIA device was only 3 bits long, while the MM ROSA has bursts of length 100 bits occurring. We have therefore used the results from this test to show that no uncorrectable errors occur in the GBTIA ROSA.

\section{DISCUSSION}

One of the aims of this test was to attempt and understand the physical mechanisms responsible for producing: single bit errors, short bursts, long bursts, and the 1-0 bit transitions which were observed for the first time in photoreceiver by [8]. Using the information on the error statistics detailed above, along with the results of the in-lab error simulator discussed below, the following conclusions on the physical mechanisms behind the different types of errors discussed above can me made.

\section{A. Origins of Burst Errors}

Long bursts only occurred in devices where the TIA was contained in the same optical package as the photodiode and was therefore also susceptible to particle strikes. Unlike single and short burst errors, long bursts were independent of both OMA and angle of incidence of the beam-which indicated to us that they could not be caused by direct ionization. In addition, they displayed no dependance on the transmitted data rate and therefore they could not have been caused by the amplifiers response to the large input signal caused by an SEU in the photodiode.

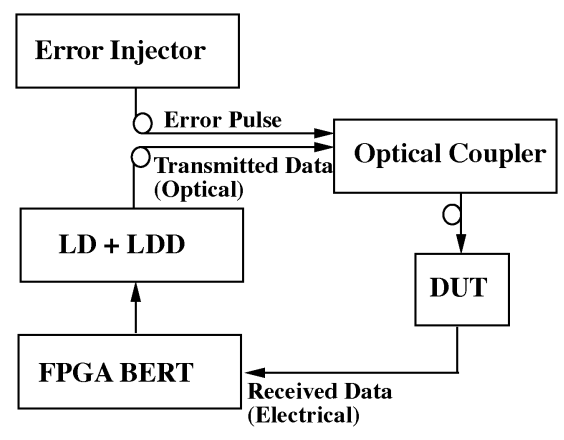

Fig. 12. Schematic of set-up used for error injector.

All of the above, combined with their low occupancy, 100\% 0-1 fraction, and our inability to reproduce them in the lab, cause us to conclude that long bursts were caused by protons striking the powering node of the amplifier, causing it to latch at high and leading to bursts of low occupancy and 100\% 0-1 fraction.

Single and short bursts are believed to be caused by upsets in the photodiode, while long bursts are caused by upsets in the powering circuit of the TIA. The first conclusion is in agreement with the accepted description for SEUs in the field, but we believe that the second is more novel and of interest to other applications were optical links are used.

\section{B. Origin of 1-0 Bit Transitions}

Previous work [8] hypothesized that 1-0 error were caused by the amplifier's response to a large signal in the photoreceiver but were unable to confirm this because they lacked access to precise simulations of the TIA from the manufacturers. We were able to use an in-lab error injector to simulate the TIAs response to large optical pulses similar to those produced by SETs.

Fig. 12 shows a schematic of the set-up used to inject errors into the GBTIA ROSA in the lab. A short $(\approx 80$ ps FWHM) optical pulse is produced using a high-speed pattern generator and a SM laser and laser driver. This short optical pulse, which represents the energy deposited by an incident particle when it strikes the sensitive volume of the device, is optically coupled to the data transmitted by the same FPGA-based BERT used in the SEU test. Both the amplitude and the position of the error pulse within a transmitted bit period could be varied using this set-up.

Fig. 13(a) shows an "SET" like pulse, generated by the error injector, superimposed on the transmitted data pattern. This set-up was used to record the amplifiers response to SET-like events, an example of which is shown in Fig. 13(b). It is clear from this that the amplifier "switches off" for a short period of time in response to this large signal.

The set-up was also used to measure the percentage of $0-1$ errors induced in the amplifier was measured as a function of the position of the error pulse within a bit period (shown here in Fig. 14).

Fig. 14 shows that the probability of a bit error being a 1-0 transition is very close to 0 , except when the error occurs near a transition between two bits. We can explain this using the response of the amplifier we measured in Fig. 13(b). When the error pulse occurs near a bit transition, and the time for the amplifier to recover is longer than that to the next bit period, then 


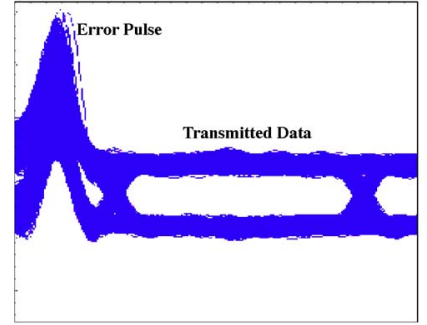

(a)

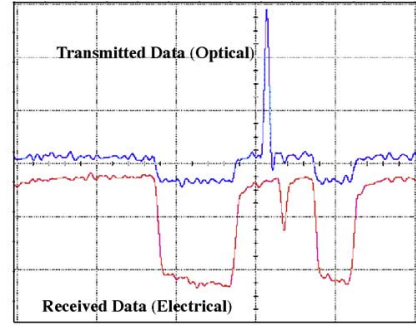

(b)
Fig. 13. Demonstrating a 1-0 bit transition in the GBTIA ROSA at $2.0 \mathrm{~Gb} / \mathrm{s}$. (a) Position of error pulse with respect to the transmitted data. (b) The amplifier's response to a large signal near a bit transition.

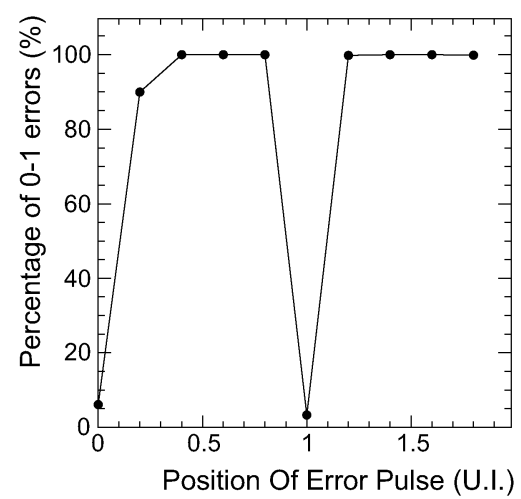

Fig. 14. Percentage of $0-1$ bit errors as a function of position of the error within a bit period in the GBTIA ROSA at $2.0 \mathrm{~Gb} / \mathrm{s}$.

the next transmitted bit will always be seen as a " 0 "—-regardless of what was transmitted. In other words, an error is only detected in the next bit if the transmitted bit was initially a " 1 "- - which is why the probability of inducing a 1-0 bit transition becomes so high.

Thus we have proven what previous work has only hypothesized [8]: that 1-0 bit transitions occur due to the amplifiers response to a large input signal caused in the receiver by an SET.

\section{CONCLUSION}

An SEU test was conducted to evaluate the performance of a candidate receiver for use in HL-LHC data transmission links. The receiver, the GBTIA ROSA, was shown to operate error free in a proton beam with a particle flux of $1.8 \times 10^{8} \mathrm{p} / \mathrm{cm}^{2} / \mathrm{s}$. In addition, the error correction scheme proposed for use in the final application of the receiver was shown to be capable of mitigating any bursts occurring in the device.

We believe that we have reached a better understanding of the physical mechanisms behind the different types of multibit errors observed in the photoreceivers tested. Single and short (at most 5 bits long) burst errors are believed to be caused by upsets in the photodiode, while those longer than 5 bits long are caused by upsets in the powering circuit of the TIA. The origin of 1-0 bit errors was also explained using an in-lab error injector. The error injector has shown that 1-0 bit transitions occur as a result of the amplifier's response time to the large input signal caused by an SEU.

Finally, previous SEU tests have suggested that the response of the amplifier chosen for a component may be tailored to provide immunity to long bursts. Here we can confirm that with knowledge of the types of errors occurring in a device, the long bursts previously observed in ROSAs, this can be done (no long bursts occurred in the custom designed GBTIA ROSA). In addition, we now have a method of understanding some aspects of the effects SEUs can have on receivers, using the in-lab error injector, without the expense and time required to set-up an in-beam test.

\section{ACKNOWLEDGMENT}

The authors would like to thank Dr. W. Hajdas and K. Egli for their help during irradiation at PSI. As well as ACEOLE, FP7, and the Marie Curie Actions for their involvement with the project.

\section{REFERENCES}

[1] N. Hessey, "Overview and electronics needs of ATLAS and CMS high luminosity upgrade," in Proc. Top. Workshop Electron. Particle Phys., Naxos, Greece, Sep. 2008, pp. 323-327.

[2] L. Amaral, S. Dris, A. Gerardin, T. Huffman, C. Issever, A. Pacheco, M. Jones, S. Kwan, S. Lee, Z. Liang, T. Liu, Z. Meng, A. Prosser, S. Padadopoulos, I. Papakonstanstinou, C. Sigaud, C. Silva, C. Soos, P. Stejskal, J. Troska, F. Vasey, P. Vichoudis, T. Weidberg, A. Xiang, and J. Yee, "The versatile link, a common project for Super-LHC," JINST, vol. 4, Dec. 2009.

[3] P. Moreira, R. Ballabriga, S. Baron, S. Bonacini, O. Cobanoglu, F. Faccio, T. Fedorov, R. Francisco, P. Gui, P. Hartin, K. Kloukinas, X. Llopart, A. Marchioro, C. Paillard, N. Pinilla, K. Wyllie, and B. Yu, "The GBT project," in Proc. Top. Workshop Electron. Particle Phys., Paris, France, Sep. 2009, pp. 342-346.

[4] M. Menouni, P. Gui, and P. Moreira, "The GBTIA, a 5 Gbit/s radiationhard optical receiver for the SLHC upgrades," in Proc. Top. Workshop Electron. Particle Phys., Paris, France, Sep. 2009, pp. 326-330.

[5] P. Marshall, C. Dale, and K. LaBel, "Space radiation effects in high performance fiber optic data links for satellite data management," IEEE Trans. Nucl. Sci., vol. 43, no. 2, pp. 645-653, Apr. 1996.

[6] F. Faccio, G. Berger, K. Gill, M. Huhtinen, A. Marchioro, P. Moreira, and F. Vasey, "Single event upset tests of an $80-\mathrm{Mb} / \mathrm{s}$ optical receiver," IEEE Trans. Nucl. Sci., vol. 48, no. 5, pp. 1700-1707, Oct. 2001.

[7] P. Marshall, C. Dale, M. Carts, and K. LaBel, "Particle-induced bit errors in high performance fiber optic data links for satellite data management," IEEE Trans. Nucl. Sci., vol. 41, no. 6, pp. 1958-1965, Dec. 1994.

[8] A. Pacheco, J. Troska, L. Amaral, S. Dris, D. Ricci, C. Sigaud, F. Vasey, and P. Vichoudis, "Single-event upsets in photoreceivers for multi-Gb/s data transmission," IEEE Trans. Nucl. Sci., vol. 56, no. 4, pp. 1978-1986, Aug. 2009.

[9] W. Hajdas, L. Adams, B. Nickson, and A. Zehnder, "The proton irradiation facility at the paul scherrer institute," Nucl. Instrum. Methods Phys. Res. B, vol. 113, no. 1-4, pp. 54-58, 1996.

[10] S. Detraz, S. Silva, P. Moreira, S. Papadopoulos, I. Papakonstantinou, S. Seif El Nasr, C. Sigaud, C. Soos, P. Stejskal, J. Troska, and H. Versmissen, "FPGA-based bit-error-rate tester for SEU-hardened optical links," in Proc. Top. Workshop Electron. Particle Phys., Paris, France, Sep. 2009, pp. 636-640.

[11] K. LaBel, E. Stassinopoulos, and G. Brucker, "Transient SEUs in a fiber optic system for space applications," IEEE Trans. Nucl. Sci., vol. 38, no. 6, pp. 1546-1550, Dec. 1991.

[12] E. Newcombe and S. Pasupathy, "Error rate monitoring for digital communications," Proc. IEEE, vol. 70, no. 8, pp. 805-828, Aug. 1982

[13] D. J. Kennedy and M. B. Nakhla, "Burst error characterization of FEC coded digital channels," Int. J. Satellite Commun., vol. 10, no. 5, pp. 243-250, 1992. 